ARTICLE

\title{
An antisite defect mechanism for room temperature ferroelectricity in orthoferrites
}

\author{
Shuai Ning (10 1,2凶${ }^{\text {, Abinash Kumar }}{ }^{1}$, Konstantin Klyukin (10 ${ }^{1}$, Eunsoo Cho ${ }^{1}$, Jong Heon Kim ${ }^{3}$, Tingyu Su${ }^{1}$, \\ Hyun-Suk Kim (1) ${ }^{3}$, James M. LeBeau (1) ${ }^{1}$, Bilge Yildiz (i) ${ }^{1,4} \&$ Caroline A. Ross (iD) ${ }^{1 \times}$
}

Single-phase multiferroic materials that allow the coexistence of ferroelectric and magnetic ordering above room temperature are highly desirable, motivating an ongoing search for mechanisms for unconventional ferroelectricity in magnetic oxides. Here, we report an antisite defect mechanism for room temperature ferroelectricity in epitaxial thin films of yttrium orthoferrite, $\mathrm{YFeO}_{3}$, a perovskite-structured canted antiferromagnet. A combination of piezoresponse force microscopy, atomically resolved elemental mapping with aberration corrected scanning transmission electron microscopy and density functional theory calculations reveals that the presence of $\mathrm{Y}_{\mathrm{Fe}}$ antisite defects facilitates a non-centrosymmetric distortion promoting ferroelectricity. This mechanism is predicted to work analogously for other rare earth orthoferrites, with a dependence of the polarization on the radius of the rare earth cation. Our work uncovers the distinctive role of antisite defects in providing a mechanism for ferroelectricity in a range of magnetic orthoferrites and further augments the functionality of this family of complex oxides for multiferroic applications.

\footnotetext{
${ }^{1}$ Department of Materials Science and Engineering, Massachusetts Institute of Technology, Cambridge, MA, USA. ${ }^{2}$ School of Materials Science and Engineering, National Institute for Advanced Materials, Nankai University, Tianjin, People's Republic of China. ${ }^{3}$ Department of Materials Science and Engineering, Chungnam National University, Daejeon, Korea. ${ }^{4}$ Department of Nuclear Science and Engineering, Massachusetts Institute of Technology,

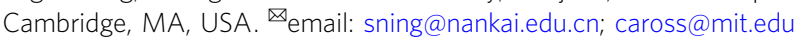


M ultiferroic materials combine ferroelectric and magnetic properties, and hold compelling interest for magnetoelectric and spintronic applications ${ }^{1}$. It has traditionally been a challenge for ferroelectricity and ferromagnetism to coexist in a single-phase material, including the perovskite-structured $\left(\mathrm{ABO}_{3}\right)$ transition metal oxides ${ }^{2}$. While magnetoelectric coupling can be artificially realized by integrating ferroelectric and magnetic phases into composites or heterostructures ${ }^{3}$, single-phase multiferroic materials and mechanisms offer substantial fundamental and technological interest, and are highly desirable for practical applications.

One of the best-known single-phase multiferroics is $\mathrm{BiFeO}_{3}$ (ref. $\left.{ }^{4}\right)$, which possesses large spontaneous polarization $(\sim 100$ $\mu \mathrm{C} / \mathrm{cm}^{2}$ ) driven by the stereochemical activity of lone-pair electrons of $\mathrm{A}$-site $\mathrm{Bi}^{3+}$ ions and weak magnetization, due to the canted antiferromagnetic ordering of $\mathrm{B}$-site $\mathrm{Fe}^{3+}$ ions ${ }^{5,6}$. Beyond this material, novel mechanisms for multiferroicity have been established by incorporating unconventional ferroelectricity into magnetic oxides ${ }^{7}$, such as the geometrically induced ferroelectricity due to polyhedral tilt and rotation in hexagonal manganites ${ }^{8,9}$ and ferrites ${ }^{10,11}$; electronically driven ferroelectricity due to charge ordering ${ }^{12,13}$; and magnetically induced ferroelectricity due to types of non-centrosymmetric spin ordering ${ }^{14}$. Strain engineering is also predicted to promote ferroelectricity in certain magnetic oxides, e.g., $\mathrm{EuTiO}_{3}$ (refs. ${ }^{15,16}$ ) and $\mathrm{SrMnO}_{3}$ (refs. ${ }^{17,18}$ ), by spin-lattice coupling.

Among such approaches, magnetically driven ferroelectricity is of great interest due to the inherent coupling expected between the ferroelectric and magnetic orderings. It was first reported in $\mathrm{TbMnO}_{3}$ that inversion symmetry breaking is caused by a cycloidal spiral spin structure at cryogenic temperatures ${ }^{19}$. Subsequently, ferroelectricity with a weak polarization $\left(0.1 \mu \mathrm{C} / \mathrm{cm}^{2}\right)$ was also observed in a family of rare-earth orthoferrites $\left(\mathrm{RFeO}_{3}\right)$ with much simpler collinear spin structures, e.g., $\mathrm{GdFeO}_{3}$ (ref. ${ }^{20}$ ) and $\mathrm{DyFeO}_{3}$ (ref. ${ }^{21}$ ). The mechanism is attributed to the exchange-striction effect of $\mathrm{R} 4 f$ and $\mathrm{Fe} 3 d$ spins, and hence the ferroelectricity only occurs below the $\mathrm{R} 4 f$ spin ordering temperature of a few Kelvin. In $\mathrm{HoFeO}_{3}$ (ref. ${ }^{22}$ ) and $\mathrm{SmFeO}_{3}$ (ref. ${ }^{23}$ ), ferroelectricity is reported to be present near or even above room temperature, but contradictory conclusions from theoretical ${ }^{24}$ and experimental ${ }^{25,26}$ work have obscured the mechanism, particularly in the case of room temperature ferroelectricity reported in $\mathrm{YFeO}_{3}(\mathrm{YFO})^{27-30}$.

YFO adopts a space group Pbnm with lattice parameters of $a_{\mathrm{o}}=$ $5.282 \AA, b_{\mathrm{o}}=5.595 \AA$, and $c_{\mathrm{o}}=7.605 \AA$ (the subscript o denotes the orthorhombic notation). Such a centrosymmetric structure inhibits spontaneous polarization in principle. The unexpected ferroelectricity found in YFO was initially attributed to spin-orbit coupling ${ }^{27}$, i.e., the inverse Dzyaloshinskii-Moriya interaction (DMI), as proposed for $\mathrm{SmFeO}_{3}$ (ref. ${ }^{23}$ ). However, it was shown that inverse DMI is unable to break the inversion symmetry in $\mathrm{SmFeO}_{3}$ (ref. ${ }^{24}$ ), precluding this mechanism. The ferroelectricity also cannot stem from spin exchange interactions because of the empty $4 f$ orbital of $\mathrm{Y}^{3+}$ ions. Moreover, the reported polarization of YFO varies dramatically in magnitude from bulk $(<0.01 \mu \mathrm{C} /$ $\left.\mathrm{cm}^{2}\right)^{27}$ to one thin film report $\left(\sim 10 \mu \mathrm{C} / \mathrm{cm}^{2}\right)^{28}$, challenging the interpretations of the origin of its polarization.

In this work, we select YFO as a model orthoferrite to investigate the mechanism that yields a robust, sizeable, and switchable ferroelectric polarization above room temperature, while preserving its magnetization. We find that, contrary to the fact that the ferroelectricity generally deteriorates upon the presence of cationic off-stoichiometry in incipient ferroelectric $\mathrm{CaTiO}_{3}\left(\right.$ ref. ${ }^{31}$ ), and analogous to the findings that cationic off-stoichiometry yields a ferroelectric distortion in antiferroelectric $\mathrm{PbZrO}_{3}$ (ref. ${ }^{32}$ ) or paraelectric $\mathrm{SrTiO}_{3}\left(\right.$ refs. $\left.{ }^{33,34}\right)$, the presence of $\mathrm{Y}-\mathrm{Fe}$ antisite $\left(\mathrm{Y}_{\mathrm{Fe}}\right)$ defects in Y-rich YFO plays a crucial role in facilitating a noncentrosymmetric distortion, which promotes a spontaneous polarization, but preserves the magnetic order. The polarization persists over a range of film thicknesses, Y-rich compositions, and lattice strains, and is supported by both theoretical simulations and experimental characterization. Density functional theory (DFT) calculations demonstrate that such an antisite defect mechanism is also expected for other rare-earth orthoferrites, in which the polarization depends on the radius of the rare-earth cation. Overall, our results demonstrate a cationic antisite defect mechanism for ferroelectricity in the family of orthoferrites, offering a strategy for designing single-phase multiferroics.

\section{Results}

Unexpected ferroelectricity in magnetic YFO thin films. YFO thin films ( $30 \mathrm{~nm}$ thick, Supplementary Note 1$)$ were grown on 001-oriented Nb-doped $\mathrm{SrTiO}_{3}$ (NSTO; $a=3.905 \AA$ ) substrates by pulsed laser deposition (PLD, see "Methods"), using a stoichiometric YFO ceramic target. High-resolution X-ray diffraction (XRD, Fig. 1a) reveals that the YFO film exhibits an epitaxial perovskite structure, while the asymmetric (013) reciprocal space mapping (RSM, Fig. 1b) shows it is not fully strained to the substrate. The broadening of the RSM peak suggests that imperfections, such as mosaicity exist. With the YFO lattice described using a pseudocubic unit cell (Fig. 1c), the in-plane $\left(a_{\mathrm{p}}\right.$, the subscript $\mathrm{p}$ denotes the pseudocubic notation) and outof-plane $\left(c_{\mathrm{p}}\right)$ lattice parameters of the YFO film are 3.862 and $3.813 \AA$, respectively, both of which are larger than the bulk values $\left(a_{\mathrm{p}}\right.$, bulk $=\frac{1}{2} \sqrt{a_{0}{ }^{2}+b_{0}{ }^{2}}=3.847 \AA, c_{\mathrm{p}}$, bulk $\left.=c_{\mathrm{o}} / 2=3.803 \AA\right)$, i.e., the unit cell volume of the thin film is larger by $+1.04 \%$ compared to bulk.

The chemical composition analyzed by high-resolution X-ray photoelectron spectroscopy (XPS) reveals that the as-prepared YFO film possesses a Y-rich composition, which is surprisingly off-stoichiometric compared to the target (Fig. 1d). XPS yielded a Y/Fe ratio of $\alpha=1.19 \pm 0.04$ for five samples prepared during several PLD runs under the same deposition conditions ("Methods", Supplementary Note 1, and Supplementary Table 1). Quantitative energy-dispersive spectroscopy (EDS) elemental analysis using a Y:Fe standard was conducted on a crosssection of one sample of $\mathrm{YFO} / \mathrm{NSTO}$ with $\mathrm{Y} / \mathrm{Fe}$ ratio $\alpha=1.19$, using scanning transmission electron microscopy (STEM). EDS reveals a uniform composition within the sample with $\mathrm{Y} / \mathrm{Fe}$ ratio of $1.21 \pm 0.12$, further corroborating the Y-rich nature of the films (Supplementary Note 1). This cationic off-stoichiometry can arise from differences in ablation or scattering rate of $\mathrm{Y}$ and Fe during the PLD process ${ }^{35}$.

Atomically resolved STEM EDS (Fig. 1e) collected on the YFO/ NSTO sample with $\alpha=1.19$ reveals the presence of $\mathrm{Y}$ ions at $\mathrm{Fe}$ sites, i.e., $\mathrm{Y}_{\mathrm{Fe}}$ defects. Based on the composition and the lamella thickness $(11 \mathrm{~nm})$, two to three antisite defects on average are expected in each $\mathrm{Fe}-\mathrm{O}$ atom column. While the STEM EDS does not have the sensitivity to resolve such small numbers of antisite defects, significant EDS intensity in the $\mathrm{Y}$ elemental map is observed for those $\mathrm{Fe}$ atom columns containing a statistically larger number of $\mathrm{Y}_{\mathrm{Fe}}$ defects. Further analysis of the simultaneously acquired, atomic number (Z)-sensitive high-angle annular dark-field (HAADF) STEM reveals an increase in atom column intensity of $10-20 \%$ at these locations, confirming the presence of $\mathrm{Y}(Z=39)$ in the $\mathrm{Fe}(Z=26)$ sites. Soft X-ray absorption spectroscopy (XAS) analysis at both the Fe $L$-edge and $\mathrm{O} K$-edge shows that $\mathrm{Fe}$ primarily adopts a +3 valence state (Supplementary Fig. 2). Given that both Fe and Y exist as trivalent ions, deviation from the ideal cation stoichiometry $\alpha=1$ can be accommodated without changes in the oxygen content. 

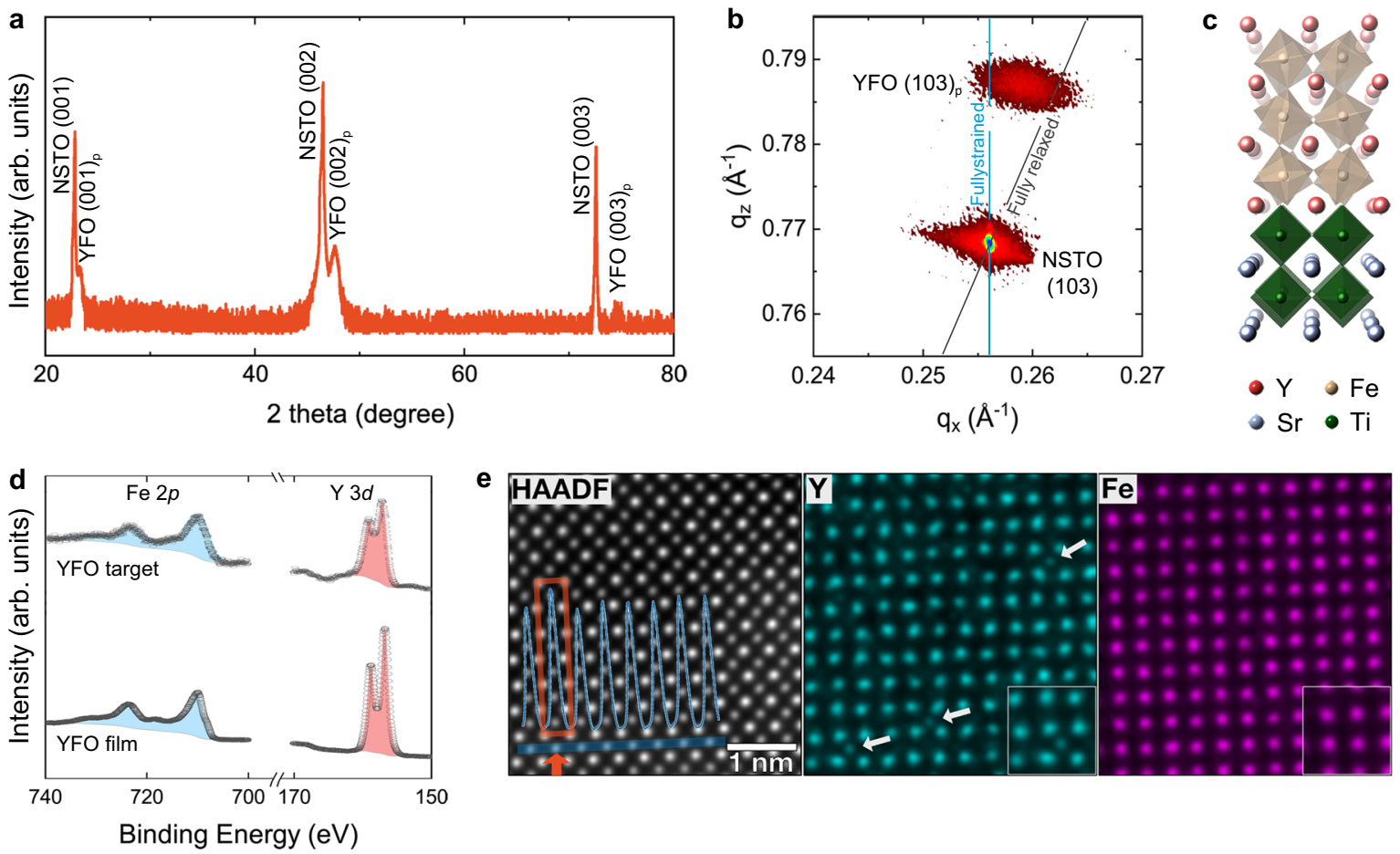

Binding Energy (eV)

Fig. 1 Structure and defect analysis of YFO thin films on NSTO substrate. a, b Structural characterization of the as-grown YFO/NSTO by high-resolution XRD (a) and asymmetric RSM (b). The subscript $p$ denotes the pseudocubic unit cell. c Schematic of lattice structures of YFO and NSTO viewed along the orthorhombic [110] axis (pseudocubic [100] axis) of the $\mathrm{YFeO}_{3}$ unit cell. d High-resolution Fe $2 p$ and $\mathrm{Y}$ 3d core level spectra of the stoichiometric YFO target and the as-prepared YFO film. e HAADF STEM image and denoised atomic resolution STEM EDS elemental mapping. The intensity profile superposed in the HAADF STEM image is taken along the blue line. Fe-O atom columns rich in $\mathrm{Y}_{\mathrm{Fe}}$ defects show increased intensity in HAADF (red arrow) and a signal in the $Y$ map (white arrows).

Bulk YFO is a perovskite-structured antiferromagnet below its Néel temperature $\left(T_{\mathrm{N}}\right)$ of $645 \mathrm{~K}$ with a Bertaut notation spin structure of $\mathrm{G}_{x} \mathrm{~A}_{y} \mathrm{~F}_{z}$, possessing a small net magnetization parallel to the $c_{0}$-axis as a result of the canting of the $\mathrm{Fe}^{3+}$ moments ${ }^{36}$. Magnetometry analysis of a YFO/NSTO sample with $\alpha=1.19$ (Fig. 2a) shows a hysteretic response along the out-of-plane direction with a saturation magnetization $\left(M_{\mathrm{s}}\right)$ of $\sim 0.056 \mu_{\mathrm{B}} / \mathrm{f}$.u., comparable with that of bulk ${ }^{37}$, and indicating that the out-ofplane orientation is the orthorhombic $c_{\mathrm{o}}$-axis. In contrast, the inplane loop is not saturated at a field of $20 \mathrm{kOe}$. Anisotropic hysteresis loops are also reported in bulk single-crystal $\mathrm{YFO}^{38}$.

Contrary to the nonferroelectric nature of bulk YFO, a sizeable ferroelectric polarization is observed at room temperature in the YFO/NSTO $(\alpha=1.19)$, as revealed by the polarization-electric field $(P-E)$ hysteresis loop (Fig. 2b). Positive-up-negative-down (PUND) pulse measurements are shown in Fig. 2c. The total remanent polarization $\left(P_{\mathrm{r}}{ }^{*}\right)$ of $9.5 \mu \mathrm{C} / \mathrm{cm}^{2}$ after the first pulse includes both switched charge density $\left(Q_{S w}\right)$ and leakage current, and the remanent polarization $\left(P_{\mathrm{r}} \wedge\right)$ of $2.3 \mu \mathrm{C} / \mathrm{cm}^{2}$ after the second positive pulse originates mainly from leakage current. The switched charge density, $Q_{\mathrm{sw}}=P_{\mathrm{r}}{ }^{*}-P_{\mathrm{r}} \wedge=7.2 \mu \mathrm{C} / \mathrm{cm}^{2}$ indicates an intrinsic and switchable remanent polarization. The polarization observed here is orders of magnitude stronger than the values reported previously in bulk $\mathrm{RFeO}_{3}, \mathrm{R}=\mathrm{Gd}^{20}, \mathrm{Dy}^{21}, \mathrm{Ho}^{22}$, and $\mathrm{Sm}^{23}$, and comparable with a measurement reported by Shang et al. in thin film $\mathrm{YFO}^{28}$.

The ferroelectric nature is confirmed by switching spectroscopy piezoresponse force microscopy (SS-PFM). The vertical PFM phase hysteresis loop and the "butterfly-shape" amplitude curve (Fig. 2d) and the piezoresponse hysteresis (Supplementary Fig. 3d) demonstrate clear reversal. This switching behavior does not qualitatively depend on the film thickness within the range of
$10-100 \mathrm{~nm}$, indicating that is it not greatly affected by strain relaxation, and persists at elevated temperature up to at least $150^{\circ}$ C, the limit of the instrument (Supplementary Fig. 4). Box-in-box writing with DC voltages of $\pm 8 \mathrm{~V}$ as shown in Fig. 2e results in $180^{\circ}$ phase contrast in the vertical PFM phase image (Fig. 2g), indicating a complete switching of polarization between upward and downward orientations. Scanning Kelvin probe force microscopy (SKPFM) was performed to exclude the possibility that the PFM signal originates from nonferroelectric mechanisms (Supplementary Note 2).

YFO was also grown on $\mathrm{SrRuO}_{3}(\mathrm{SRO}, \sim 10 \mathrm{~nm}$ thick)-buffered STO substrates. YFO/SRO/STO adopts a similar crystal structure and Y-rich stoichiometry as YFO/NSTO. Almost the same ferroelectric switching behaviors are found whether the cantilever in PFM is placed directly on the YFO film surface or on a Pt top electrode (Fig. 2h-j), further excluding surface and interface artifacts. We also prepared YFO films on different substrates with conductive layers (see "Methods"), including SRO-buffered $\mathrm{DyScO}_{3}\left(\mathrm{DSO}, a_{\mathrm{p}}=3.944 \AA\right.$ ), $\mathrm{La}_{0.67} \mathrm{Sr}_{0.33} \mathrm{MnO}_{3}$ (LSMO)-buffered $\left(\mathrm{LaAlO}_{3}\right)_{0.3}-\left(\mathrm{SrAl}_{0.5} \mathrm{Ta}_{0.5} \mathrm{O}_{3}\right)_{0.7} \quad\left(\mathrm{LSAT}, \quad \alpha_{\mathrm{p}}=3.868 \AA\right), \quad$ and LSMO-buffered $\mathrm{LaAlO}_{3}$ (LAO, $\alpha_{\mathrm{p}}=3.788 \AA$ ). Again, Y-rich stoichiometry and ferroelectric switching are consistently observed across these samples (Supplementary Fig. 6), but a small dependence of the remanent piezoresponse on the substrate lattice parameter (Supplementary Table 2) suggests that the epitaxial strain plays a minor role in the piezoresponse.

Dependence of ferroelectricity on the cation stoichiometry. The effects of cation stoichiometry on the ferroelectricity were first evaluated by growing a sample using a $\mathrm{Y}_{3} \mathrm{Fe}_{5} \mathrm{O}_{12}$ (yttrium iron garnet, YIG) target. High-resolution XPS suggests that the as- 
a

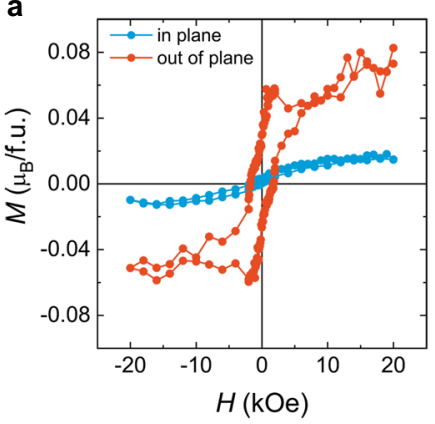

d

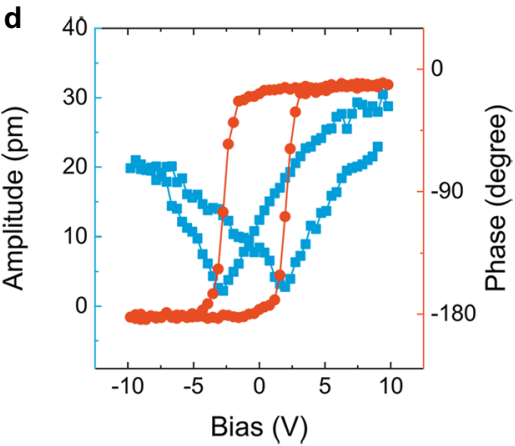

h

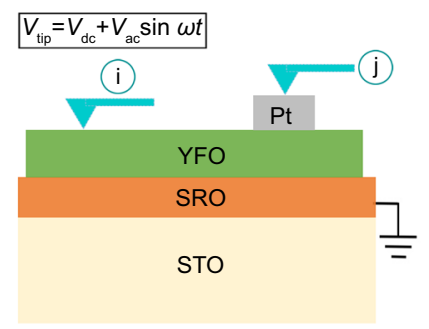

b

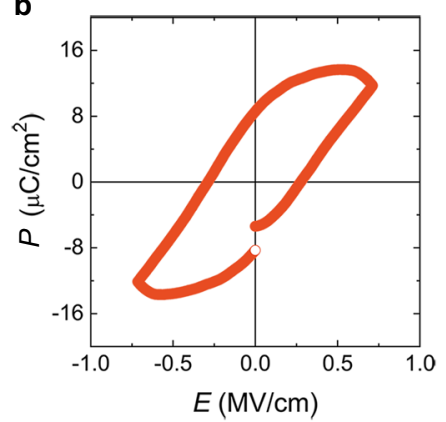

C

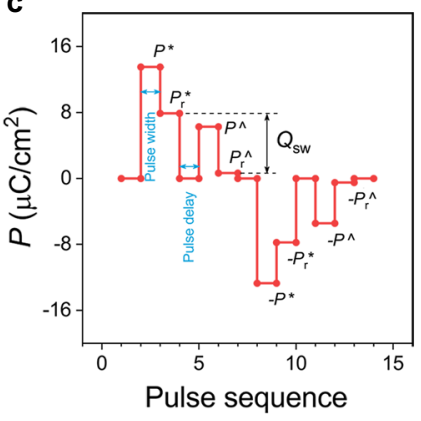

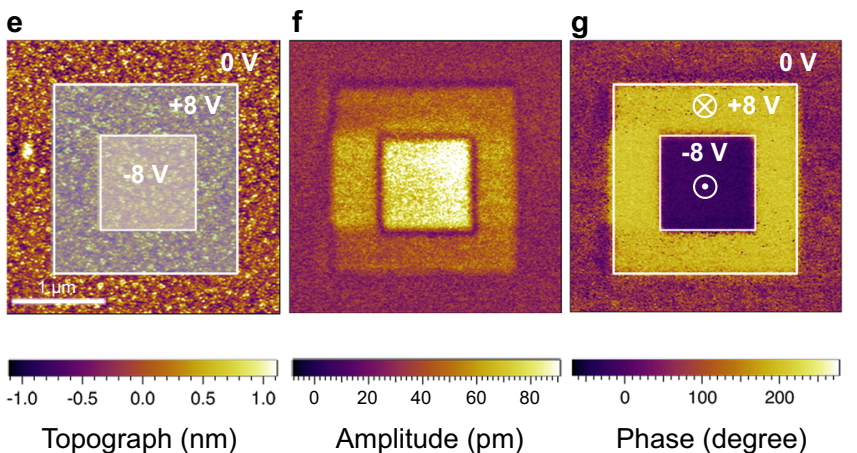
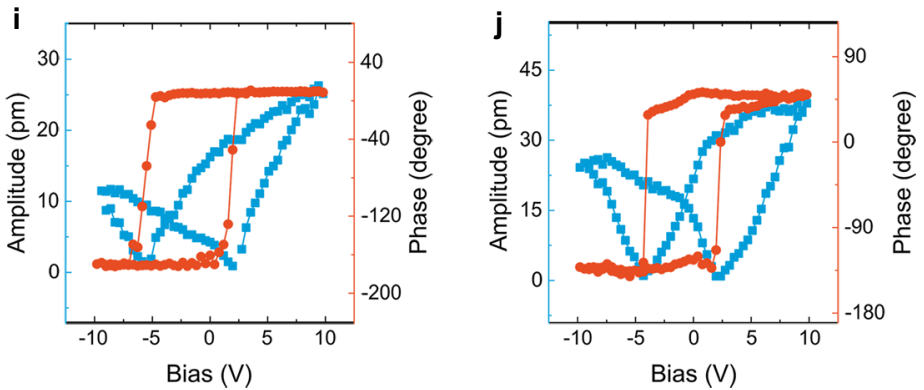

Fig. 2 Magnetic and ferroelectric properties of YFO thin films. a $M-H$ curves measured at $5 \mathrm{~K}$. b Macroscopic $P-E$ loop measured at room temperature with a frequency of $50 \mathrm{~Hz}$. c PUND measurements with pulse width of $1 \mathrm{~ms}$ and pulse delay of $100 \mathrm{~ms}$. Q $\mathrm{Sw}_{\mathrm{s}}$ is the switched charge density. $\mathbf{d}$ Local SS-PFM amplitude curve and phase loop. e-g Box-in-box writing experiments carried out as indicated in the topographic image (e). The vertical PFM amplitude (f) and vertical PFM phase (g) images are collected subsequently. $\mathbf{h}$ Cross-sectional schematic of SS-PFM measurements with the cantilever loaded on the surface of YFO film or on the Pt electrode. $\mathbf{i}, \mathbf{j}$ Local SS-PFM amplitude curves and phase loops collected with the cantilever on the YFO film (i) and on the top of Pt electrode $(\mathbf{j})$, respectively.

prepared "YIG"-composition thin film on NSTO substrate shows almost the same $\mathrm{Y} / \mathrm{Fe}$ ratio as the target, i.e., $\alpha=0.6$, while the STEM EDS analysis indicates compositional heterogeneity with regions having relative excess of $\mathrm{Y}$ or $\mathrm{Fe}$, with respect to the average $\alpha=0.6$ (Supplementary Note 5). The film exhibits a perovskite structure (Fig. 3a), which is qualitatively different from the garnet structure of bulk YIG. A series of $\mathrm{Y}_{\alpha} \mathrm{FeO}_{1.5(\alpha+1)}(0.60$ $<\alpha<1.19$ ) thin films were then prepared on NSTO by codeposition from the YFO and YIG targets. All of the films adopt the perovskite structure (Supplementary Fig. 7b).

The ferroelectric behavior depends strongly on the Y/Fe ratio as revealed by SS-PFM. For the Y-deficient regime $\alpha<1$ no switching is found (Fig. $3 \mathrm{~b}, \mathrm{c}$ ), while for the Y-rich regime, i.e., $\alpha$ $=1.03$ (Fig. 3d), 1.11 (Fig. 3e), and 1.19 (Fig. 2d), consistent ferroelectric switching is observed. The out-of-plane lattice dimension gradually expands (Fig. 3a) as $\alpha$ becomes either greater or smaller than 1 , as a consequence of cation defects. Comparing samples with $\alpha=1.11$ and $\alpha=0.60$, the lattice parameters and unit cell volume are almost the same, but the ferroelectric response is markedly different, pointing to composition as the key factor. Moreover, as $\alpha$ decreases, the remanent piezoresponse gradually weakens (Supplementary Fig. 7i). We therefore conclude that the $\mathrm{Y} / \mathrm{Fe}$ stoichiometry plays the dominant role in the room temperature ferroelectricity of epitaxial YFO thin films.

Mechanism of ferroelectricity induced by $\mathrm{Y}_{\mathrm{Fe}}$ defects. To explain the physical basis of these experimental results, firstprinciples DFT simulations were employed to calculate the electronic structure and atomic positions of Y-rich YFO (see "Methods"). The formation energy of various types of point defects in the perovskite YFO lattice evaluated with appropriate chemical potentials for each element suggests that the $\mathrm{Y}_{\mathrm{Fe}}$ (or $\mathrm{Fe}_{\mathrm{Y}}$ ) antisite defects have much lower formation energies than cation vacancies (Supplementary Note 6), which is consistent with the result of first-principles calculations in $\mathrm{YIG}^{39}$ and the presence of $\mathrm{Y}_{\mathrm{Fe}}$ in our as-prepared Y-rich YFO film. The $\mathrm{Y}_{\mathrm{Fe}}$ defects are stabilized by tensile in-plane strain, and are unlikely to migrate upon the application of moderate external voltages because of a high migration barrier of $3.1 \mathrm{eV}$ (Supplementary Note 6). 

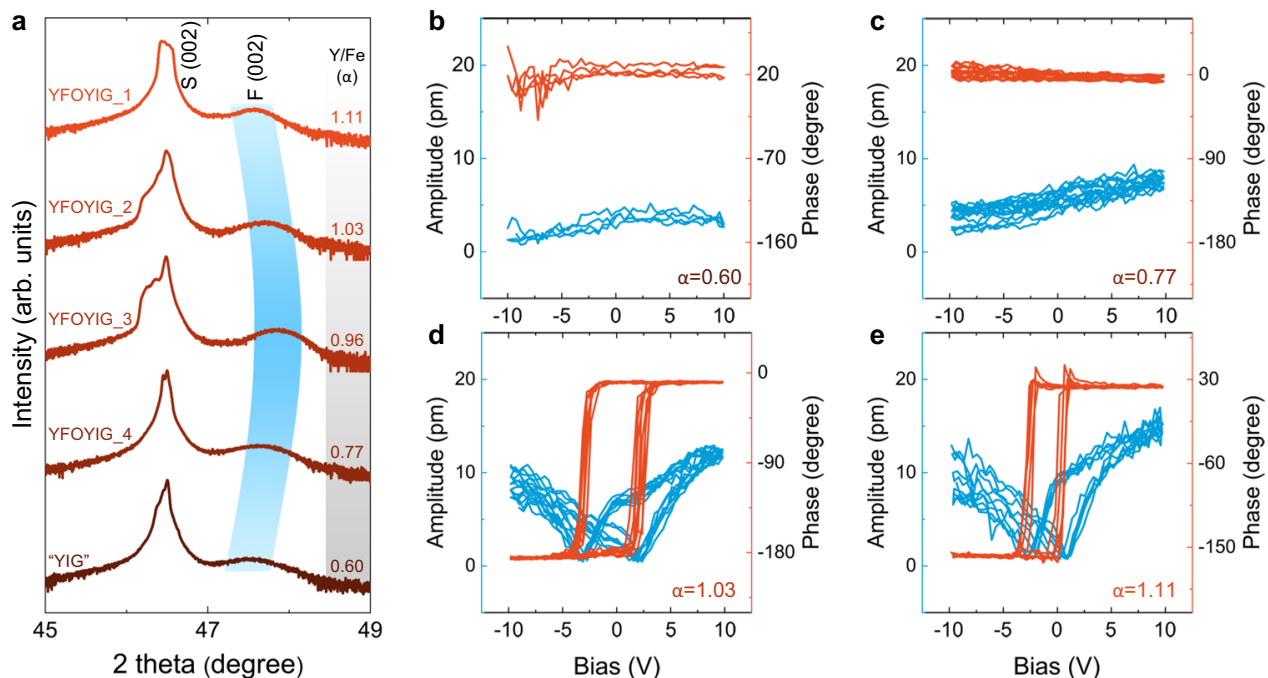

Fig. 3 Dependence of structure and ferroelectric switching on the $\mathbf{Y} / \mathbf{F e}$ ratio. a High-resolution XRD pattern of the 002 family of peaks of the film deposited from the YIG target and co-deposited $\mathrm{Y}_{\alpha} \mathrm{FeO}_{1.5(\alpha+1)}$ films grown on NSTO substrates. $\mathrm{S}$ and $\mathrm{F}$ refer to the peaks of substrate and film. The $\mathrm{Y} / \mathrm{Fe}$ ratio, i.e., $\alpha$, determined by high-resolution XPS analysis is labeled correspondingly. b-e Local SS-PFM amplitude curves and phase loops of "YIG" films ( $\alpha$ $=0.60)$ (b) and co-deposited $\mathrm{Y}_{\alpha} \mathrm{FeO}_{1.5(\alpha+1)}$ thin films with $\alpha=0.77(\mathbf{c}), 1.03$ (d), and 1.11 (e), respectively.
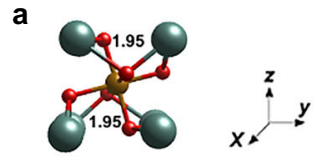

b

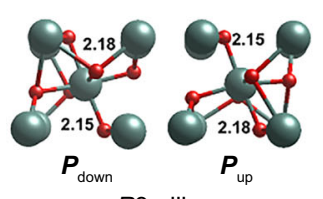

R3c-like

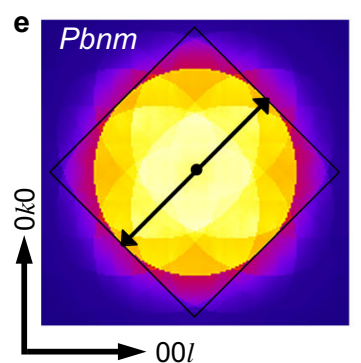

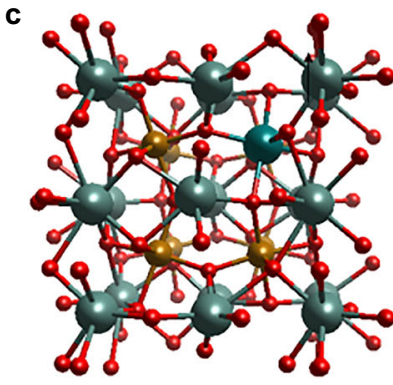

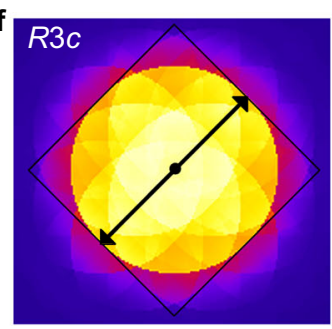

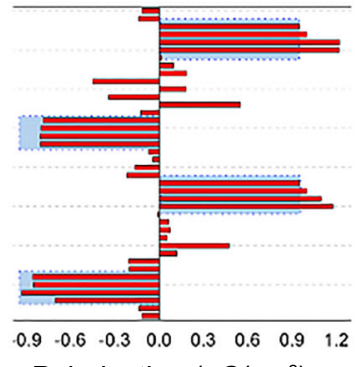

Polarization $\left(\mu \mathrm{C} / \mathrm{cm}^{2}\right)$

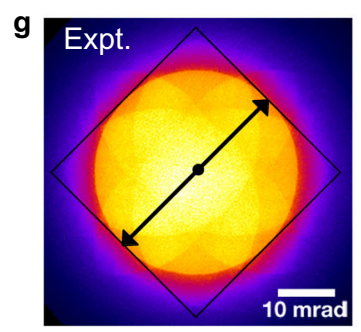

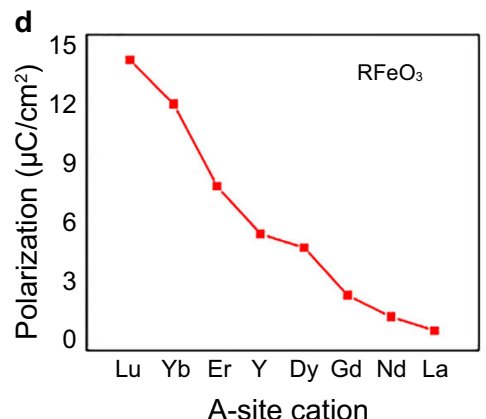

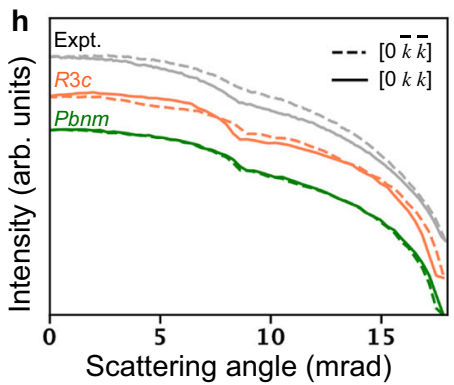

Fig. 4 Crystallographic origin of ferroelectricity in Y-rich YFO. a, b Schematics of centrosymmetric Pbnm (a) and non-centrosymmetric R3c-like (b) structures. c Crystal structure and layer-resolved polarization for Y-rich YFO. Red columns indicate the atomic contribution to the ferroelectric polarization in Y-rich YFO calculated using the Born charge approximation, while blue columns show layer-resolved contributions to the ferroelectric polarization in stoichiometric YFO. d Ferroelectric polarization for various orthoferrites $\mathrm{RFeO}_{3}$ that are assumed to be epitaxially grown on $\mathrm{SrTiO}_{3}$ for all calculations with $\mathrm{R} / \mathrm{Fe}=1.28$. e-g PACBED patterns including the simulated patterns, using the DFT relaxed centrosymmetric Pbnm (e) and non-centrosymmetric $R 3 \mathrm{C}$ (f) and the experimental result measured from the Y-rich YFO $(\alpha=1.19)$ thin film sample $(\mathbf{g})$. $\mathbf{h}$ Intensity profiles integrated across the pattern diagonals from PACBED patterns along the black arrows shown in e-g.

The symmetry calculation suggests the origin of the ferroelectricity lies in the structural distortion around the $\mathrm{Y}_{\mathrm{Fe}}$ sites, where the Pbnm structure (Fig. 4a) is locally distorted into a region with $R 3 c$-like symmetry (Fig. 4b). In YFO, the changes in bonding of oxygen atoms adjacent to $\mathrm{Y}_{\mathrm{Fe}}$ break the inversion symmetry, generating a net local dipole moment (Fig. 4b), which further polarizes the surrounding stoichiometric regions, producing a spontaneous polarization. The layer-resolved polarization for Yrich YFO, calculated using the Born charge approximation, is given in Fig. 4c. Both $\mathrm{Y}_{\mathrm{Fe}}$ defects and epitaxial strain promote the formation of the $R 3 c$ regions within the Pbnm YFO structure (Supplementary Note 7).

Position-averaged convergent beam electron diffraction (PACBED), which is sensitive to inversion center symmetry breaking ${ }^{40}$, confirms the crystallographic origins of ferroelectricity. Compared to the simulated patterns using the DFT relaxed structures of both centrosymmetric Pbnm (Fig. 4e) and noncentrosymmetric $R 3 c$ (Fig. $4 \mathrm{f}$ ), the experimental pattern (Fig. $4 \mathrm{~g}$ ) 
measured from the Y-rich YFO $(\alpha=1.19)$ film exhibits an asymmetric feature, i.e., a mismatch of intensity profiles between $\left[\begin{array}{lll}0 & k & k\end{array}\right]$ and $[0 \bar{k} \bar{k}]$ directions (Fig. 4h), which is consistent with the simulated non-centrosymmetric $R 3 c$, but different from the centrosymmetric $\mathrm{Pbnm}$ pattern where the intensity profile along $\left[\begin{array}{lll}0 & k & k\end{array}\right]$ coincides exactly with that along $[0 \bar{k} \bar{k}]$. These results support the non-centrosymmetric structure predicted by our DFT calculations and provide evidence that the $\mathrm{Y}_{\mathrm{Fe}}$ defects induce inversion symmetry breaking that can support ferroelectricity.

The DFT calculations predict a switchable out-of-plane polarization of $7.2 \mu \mathrm{C} / \mathrm{cm}^{2}$ for YFO with $\alpha=1.28$ (Supplementary Note 7), in reasonable agreement with our experimental results. The calculations also indicate that ordering of $\mathrm{Y}_{\mathrm{Fe}}$ antisites has little effect on the spontaneous polarization (Supplementary Fig. 10). $R 3 \mathrm{c}$ structures in many other $\mathrm{RFeO}_{3}$ orthoferrites with magnetic and nonmagnetic $\mathrm{A}$-site ions $(\mathrm{R}=\mathrm{Lu}$, $\mathrm{Yb}, \mathrm{Er}, \mathrm{Y}, \mathrm{Dy}, \mathrm{Gd}, \mathrm{Nb}$, and $\mathrm{La}$ ) were also modeled. The $\mathrm{R}_{\mathrm{Fe}}$ antisite defects favor ferroelectric behavior (Fig. 4d) across the series, suggesting the general nature of this mechanism to yield multiferroicity in orthoferrites. In the $R 3 c$ structure the spontaneous polarization falls monotonically with an increase of the ionic radius of the rare-earth cation. DFT further shows that the antiferromagnetic order in the YFO is robust in the presence of $\mathrm{Y}_{\mathrm{Fe}}$ (Supplementary Note 7).

To summarize, our study reveals a mechanism for ferroelectricity based on antisite defects in the perovskite-structured canted antiferromagnet $\mathrm{YFO}$ with excess $\mathrm{Y}$. The $\mathrm{Y}_{\mathrm{Fe}}$ defects play a dominant role in promoting a non-centrosymmetric structural distortion that yields a switchable polarization, which is robust above room temperature, and present in films grown with a range of strain states and film thicknesses. The antisite defects are stable and occur without changes in oxygen content because the $\mathrm{Y}$ and Fe cations are isovalent.

According to first-principles calculations, the relationship between cation off-stoichiometry and ferroelectricity is expected to occur generally within the family of orthoferrites, with magnitude depending on the specific A-site cation. Given the rich magnetic phase diagram particularly for those $\mathrm{RFeO}_{3}$, where $\mathrm{R}$ has partially filled $f$ electrons, it is of substantial interest and significance to investigate the influence of antisite defects on the magnetic properties, and the interactions between ferroelectric and magnetic orderings. Our work therefore opens a route for promoting ferroelectricity and multiferroicity in single-phase orthoferrites.

\section{Methods}

Thin film preparation. The YFO thin films were prepared by PLD using a KrF excimer laser $(\lambda=248 \mathrm{~nm})$ with $1.3 \mathrm{~J} / \mathrm{cm}^{2}$ fluence and $10 \mathrm{~Hz}$ repetition rate to ablate a ceramic YFO target. The setpoint temperature of the substrate holder was $900^{\circ} \mathrm{C}$ and the substrate itself was $\sim 100^{\circ} \mathrm{C}$ below this. The oxygen partial pressure, $p\left(\mathrm{O}_{2}\right)$, was 10 mTorr. After growth, the films were cooled down to room temperature in the same $p\left(\mathrm{O}_{2}\right)$ at a rate of $20^{\circ} \mathrm{C} / \mathrm{min}$. For those grown on nonconductive substrates, a 10-nm-thick SRO layer was grown on STO or DSO at $850^{\circ}$ $\mathrm{C}$ substrate holder temperature under $p\left(\mathrm{O}_{2}\right)=5 \mathrm{mT}$ orr, and a 15 -nm-thick LSMO layer was grown on LSAT or LAO at $800^{\circ} \mathrm{C}$ substrate holder temperature under $p$ $\left(\mathrm{O}_{2}\right)=10$ mTorr. The "YIG" thin films were grown on NSTO substrates at the same conditions by ablating a ceramic YIG target. A series of $\mathrm{Y}_{\alpha} \mathrm{FeO}_{1.5(\alpha+1)}$ thin films were prepared by codeposition using YFO and YIG targets.

Composition and structural characterization. The chemical composition is

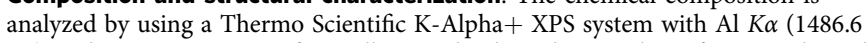
$\mathrm{eV}$ ) as the X-ray source. Before collecting the data, the sample surface was cleaned with a cluster Ar-ion beam for $30 \mathrm{~s}$. For quantitative analysis of the $\mathrm{Y} / \mathrm{Fe}$ ratio of YFO films, the stoichiometric YFO target whose Y/Fe ratio is $1: 1$ was measured, and the ratio of the integrated areas of $\mathrm{Y} 3 d$ and Fe $2 p$ core level spectra was taken as a reference. XAS measurements at $\mathrm{Fe} L$-edges and $\mathrm{O} K$-edges were performed in total electron yield (TEY) modes, using the beamline 4-ID-C of the Advanced Photon Source at Argonne National Laboratory. The temperature was set to $200 \mathrm{~K}$ to obtain a good signal to noise ratio. Reference scans of elemental Fe measured simultaneously indicate negligible energy shift throughout the experiments. The film thickness was analyzed by X-ray reflectivity, and the crystalline structure was characterized by both high-resolution XRD and RSM, using a Rigaku SmartLab high-resolution diffractometer with $\mathrm{Cu} K \alpha_{1}$ radiation $(\lambda=1.5406 \AA)$ as X-ray source and an incident beam Ge-(220) double-bounce monochromator.

Transmission electron microscopy. Cross-sectional samples of thin film YFO were prepared for electron microscopy by mechanical wedge polishing and final thinning, using cryogenic Ar-ion milling. STEM analysis was performed with a probe corrected Thermo Fisher Scientific Titan G3 $60-300 \mathrm{kV}$ operated at $200 \mathrm{kV}$ with a probe convergence semiangle of $18 \mathrm{mrad}$. A collection semiangle range of 63-200 mrad was used for STEM imaging. Atomic resolution EDS elemental maps were collected with a Thermo Fisher Scientific Super-X EDS detector, and Y and Fe elemental maps were denoised using nonlocal principal component analysis, and gaussian blurring using an open-source Matlab script ${ }^{41}$. The $\mathrm{Y} / \mathrm{Fe}$ ratio was quantified using the stoichiometric YFO target as reference. PACBED patterns were simulated with a custom Python-based STEM simulation, using the multislice approach $^{42}$.

Magnetic and ferroelectric properties measurements. Magnetic properties were analyzed using a Quantum Design MPMS-3 SQUID magnetometer and a Digital Measurement System 7035B vibrating sample magnetometer. In the SQUID magnetometer out-of-plane $M-H$ measurements were performed on a $2 \mathrm{~mm} \times 2$ $\mathrm{mm}$ YFO film sample mounted on a semicylindrical quartz holder, while in-plane measurements were performed on a $4 \mathrm{~mm} \times 4 \mathrm{~mm}$ sample, yielding a higher signal to noise ratio. A Precision Premier II Ferroelectric Tester was used to perform the $P-E$ loops and PUND measurements with a home-made probe station. SS-PFM, PFM, and SKPFM measurements were performed on a commercial atomic force microscope (Cypher, Asylum Research) under dual-frequency resonant tracking modes with Pt-coated Si conductive probes (MikroMasch, HQ:NSC18/Pt).

Density functional theory calculation. First-principles calculations using DFT were first performed to evaluate the formation energy of various point defects in YFO, using the projector augmented wave method as implemented in Vienna $\mathrm{Ab}$ initio Simulations Package ${ }^{43,44}$. The plane wave energy cutoff of $500 \mathrm{eV}$ and Monkhorst-Pack $k$-point sampling were used. The YFO unit cell is calculated using a $4 \times 4 \times 4 k$-point mesh with generalized gradient approximation for which the Perdew-Burke-Ernzerhof functional was used ${ }^{45}$. Defect calculations were performed on 80 atoms in a $2 \times 2 \times 1$ supercell (or a $3 \times 4 \times 4$ supercell for Supplementary Fig. 9) with a Monkhorst-Pack $2 \times 2 \times 2$ mesh. The total energies and forces were converged to $<10^{-6} \mathrm{eV}$ and $5 \mathrm{meV} / \AA$, respectively. Ferroelectric properties were calculated using the Berry-phase approach ${ }^{46}$. A pseudocubic $\left(\sqrt{2} a_{\mathrm{o}} \times \sqrt{2} b_{\mathrm{o}} \times c_{\mathrm{o}}\right)$ Pbnm supercell with a single $\mathrm{Y}_{\mathrm{Fe}}$ antisite defect, and fixed inplane lattice parameters was used to simulate $\mathrm{Y} / \mathrm{Fe}$ non-stoichiometry and epitaxia growth on a $\mathrm{SrTiO}_{3}$ substrate. Born effective charges approximation was used to derive atomic-resolved contributions to ferroelectric polarization. To estimate polarization switching barriers, we calculated the migration energy profile along the minimum energy path between two polarization states, using the climbing image nudged elastic band method ${ }^{47}$. The choice of exchange-correlation functional is discussed in Supplementary Note 7.

\section{Data availability}

The data that support the findings of this study are available from the corresponding author on reasonable request.

Received: 16 December 2020; Accepted: 27 June 2021; Published online: 14 July 2021

\section{References}

1. Cheong, S. W. \& Mostovoy, M. Multiferroics: a magnetic twist for ferroelectricity. Nat. Mater. 6, 13-20 (2007).

2. Hill, N. A. Why are there so few magnetic ferroelectrics? J. Phys. Chem. B 104, 6694-6709 (2000).

3. Ramesh, R. \& Spaldin, N. A. Multiferroics: progress and prospects in thin films. Nat. Mater. 6, 21-29 (2007)

4. Catalan, G. \& Scott, J. F. Physics and applications of bismuth ferrite. $A d v$. Mater. 21, 2463-2485 (2009).

5. Wang, J. et al. Epitaxial $\mathrm{BiFeO}_{3}$ multiferroic thin film heterostructures. Science 299, 1719-1722 (2003).

6. Eerenstein, W. et al. Comment on "Epitaxial $\mathrm{BiFeO}_{3}$ multiferroic thin film heterostructures”. Science 307, 1203 (2005). author reply 1203.

7. Spaldin, N. A. \& Ramesh, R. Advances in magnetoelectric multiferroics. Nat Mater. 18, 203-212 (2019). 
8. Van Aken, B. B., Palstra, T. T., Filippetti, A. \& Spaldin, N. A. The origin of ferroelectricity in magnetoelectric $\mathrm{YMnO}_{3}$. Nat. Mater. 3, 164-170 (2004).

9. Lilienblum, M. et al. Ferroelectricity in the multiferroic hexagonal manganites. Nat. Phys. 11, 1070-1073 (2015).

10. Jeong, Y. K. et al. Structurally tailored hexagonal ferroelectricity and multiferroism in epitaxial $\mathrm{YbFeO}_{3}$ thin-film heterostructures. J. Am. Chem. Soc. 134, 1450-1453 (2012).

11. Ahn, S.-J. et al. Artificially imposed hexagonal ferroelectricity in canted antiferromagnetic $\mathrm{YFeO}_{3}$ epitaxial thin films. Mater. Chem. Phys. 138, 929-936 (2013).

12. Ikeda, N. et al. Ferroelectricity from iron valence ordering in the chargefrustrated system $\mathrm{LuFe}_{2} \mathrm{O}_{4}$. Nature 436, 1136-1138 (2005).

13. Efremov, D. V., van den Brink, J. \& Khomskii, D. I. Bond-versus site-centred ordering and possible ferroelectricity in manganites. Nat. Mater. 3, 853-856 (2004).

14. Tokura, Y., Seki, S. \& Nagaosa, N. Multiferroics of spin origin. Rep. Prog. Phys. 77, 076501 (2014).

15. Fennie, C. J. \& Rabe, K. M. Magnetic and electric phase control in epitaxial $\mathrm{EuTiO}_{3}$ from first principles. Phys. Rev. Lett. 97, 267602 (2006).

16. Lee, J. H. et al. A strong ferroelectric ferromagnet created by means of spinlattice coupling. Nature 466, 954-958 (2010).

17. Lee, J. H. \& Rabe, K. M. Epitaxial-strain-induced multiferroicity in $\mathrm{SrMnO}_{3}$ from first principles. Phys. Rev. Lett. 104, 207204 (2010).

18. Guo, J. W. et al. Strain-induced ferroelectricity and spin-lattice coupling in $\mathrm{SrMnO}_{3}$ thin films. Phys. Rev. B 97, 235135 (2018).

19. Kimura, T. et al. Magnetic control of ferroelectric polarization. Nature $\mathbf{4 2 6}$, 55-58 (2003).

20. Tokunaga, Y. et al. Composite domain walls in a multiferroic perovskite ferrite. Nat. Mater. 8, 558-562 (2009).

21. Tokunaga, Y., Iguchi, S., Arima, T. \& Tokura, Y. Magnetic-field-induced ferroelectric state in $\mathrm{DyFeO}_{3}$. Phys. Rev. Lett. 101, 097205 (2008).

22. Dey, K. et al. Natural ferroelectric order near ambient temperature in the orthoferrite $\mathrm{HoFeO}_{3}$. Phys. Rev. B 100, 214432 (2019).

23. Lee, J. H. et al. Spin-canting-induced improper ferroelectricity and spontaneous magnetization reversal in $\mathrm{SmFeO}_{3}$. Phys. Rev. Lett. 107, 117201 (2011).

24. Johnson, R. D., Terada, N. \& Radaelli, P. G. Comment on "Spin-cantinginduced improper ferroelectricity and spontaneous magnetization reversal in $\mathrm{SmFeO}_{3}$ ”. Phys. Rev. Lett. 108, 219701 (2012). author reply 219702.

25. Kuo, C. Y. et al. $k=0$ magnetic structure and absence of ferroelectricity in $\mathrm{SmFeO}_{3}$. Phys. Rev. Lett. 113, 217203 (2014).

26. Cheng, Z. et al. Interface strain-induced multiferroicity in a $\mathrm{SmFeO}_{3}$ film. ACS Appl Mater. Interfaces 6, 7356-7362 (2014).

27. Shang, M. et al. The multiferroic perovskite $\mathrm{YFeO}_{3}$. Appl. Phys. Lett. 102, 062903 (2013).

28. Shang, M., Wang, C., Chen, Y., Sun, F. \& Yuan, H. The multiferroic epitaxial thin film $\mathrm{YFeO}_{3}$. Mater. Lett. 175, 23-26 (2016).

29. Shang, M. et al. Multiferroicity in the $\mathrm{YFeO}_{3}$ crystal. Funct. Mater. Lett. 13 1950088 (2020).

30. Nagrare, B. S., Kekade, S. S., Thombare, B., Reddy, R. V. \& Patil, S. I. Hyperfine interaction, Raman and magnetic study of $\mathrm{YFeO}_{3}$ nanocrystals. Solid State Commun. 280, 32-38 (2018).

31. Haislmaier, R. C. et al. Unleashing strain induced ferroelectricity in complex oxide thin films via precise stoichiometry control. Adv. Funct. Mater. 26, 7271-7279 (2016).

32. Gao, R. et al. Ferroelectricity in $\mathrm{Pb}_{1+\delta} \mathrm{ZrO}_{3}$ thin films. Chem. Mater. 29, 6544-6551 (2017).

33. Lee, D. et al. Emergence of room-temperature ferroelectricity at reduced dimensions. Science 349, 1314-1317 (2015).

34. Klyukin, K. \& Alexandrov, V. Effect of intrinsic point defects on ferroelectric polarization behavior of $\mathrm{SrTiO}_{3}$. Phys. Rev. B 95, 035301 (2017).

35. Ojeda-G-P, A., Döbeli, M. \& Lippert, T. Influence of plume properties on thin film composition in pulsed laser deposition. Adv. Mater. Interfaces 5, 1701062 (2018).

36. White, R. L. Review of recent work on the magnetic and spectroscopic properties of the rare-earth orthoferrites. J. Appl. Phys. 40, 1061-1069 (1969).

37. Jacobs, I. S., Burne, H. F. \& Levinson, L. M. Field-induced spin reorientation in $\mathrm{YFeO}_{3}$ and $\mathrm{YCrO}_{3}$. J. Appl. Phys. 42, 1631-1632 (1971).

38. Wu, A. et al. Crystal growth and magnetic property of $\mathrm{YFeO}_{3}$ crystal. Bull. Mater. Sci. 35, 259-263 (2012).

39. Tan, S., Zhang, W., Yang, L., Chen, J. \& Wang, Z. Intrinsic defects in yttrium iron garnet: a first-principles study. J. Appl. Phys. 128, 183904 (2020).

40. LeBeau, J. M., D’Alfonso, A. J., Wright, N. J., Allen, L. J. \& Stemmer, S. Determining ferroelectric polarity at the nanoscale. Appl. Phys. Lett. 98, 052904 (2011).

41. J. Salmon, C. D, R. Willett, Z. Harmany. Poisson noise reduction with nonlocal PCA. In 2012 IEEE International Conference on Acoustics, Speech and Signal Processing (ICASSP), 1109-1112 (IEEE, 2012).
42. Kirkland, E. J. Advanced Computing in Electron Microscopy (Springer, 2010),

43. Kresse, G. \& Furthmuller, J. Efficient iterative schemes for ab initio totalenergy calculations using a plane-wave basis set. Phys. Rev. B 54, 11169-11186 (1996).

44. Blöchl, P. E. Projector augmented-wave method. Phys. Rev. B 50, 17953-17979 (1994).

45. Perdew, J. P., Burke, K. \& Ernzerhof, M. Generalized gradient approximation made simple. Phys. Rev. Lett. 77, 3865-3868 (1996).

46. King-Smith, R. D. \& Vanderbilt, D. Theory of polarization of crystalline solids. Phys. Rev. B 47, 1651-1654 (1993).

47. Henkelman, G. \& Jónsson, H. Improved tangent estimate in the nudged elastic band method for finding minimum energy paths and saddle points. J. Chem. Phys. 113, 9978-9985 (2000).

\section{Acknowledgements}

The authors are grateful to Dr. Guo Tian for helpful discussions. This work was supported by the MRSEC Program of the National Science Foundation under award No. DMR-1419807. This work was performed in part at the Center for Nanoscale Systems (CNS), a member of the National Nanotechnology Infrastructure Network (NNIN), and part of Harvard University, supported by the National Science Foundation under award No. ECS-0335765. This work also used resources of the Advanced Photon Source, a US Department of Energy (DOE) Office of Science User Facility operated for the DOE Office of Science by Argonne National Laboratory under contact No. DE-AC02-06CH11357. The DFT calculations were carried out using the Extreme Science and Engineering Discovery Environment (XSEDE), which is supported by National Science Foundation Grant No. ACI-4321548562. Computational work was also partially supported by National Institute of Supercomputing and Networking/Korea Institute of Science and Technology Information with supercomputing resources, including technical support (Grant No. KSC-2019-CRE-0044). A.K. acknowledges support from MIT MathWorks Engineering Fellowships. The NVIDIA Titan Xp GPU used for this research was donated by the NVIDIA Corporation.

\section{Author contributions}

S.N. conceived the original idea and C.A.R. supervised the project. S.N. prepared the samples and characterized the structure, composition, magnetic, and ferroelectric properties with assistance from E.C. and T.S. A.K. performed the TEM measurements and PACBED analysis under supervision of J.M.L. K.K., J.H.K., H.-S.K., and B.Y. performed the DFT calculations. S.N. and C.A.R. wrote the manuscript. All the co-authors discussed the results and revised the manuscript.

\section{Competing interests}

The authors declare no competing interests.

\section{Additional information}

Supplementary information The online version contains supplementary material available at https://doi.org/10.1038/s41467-021-24592-w.

Correspondence and requests for materials should be addressed to S.N. or C.A.R.

Peer review information Nature Communications thanks Zhaosheng Li and the other, anonymous, reviewer(s) for their contribution to the peer review of this work.

Reprints and permission information is available at http://www.nature.com/reprints

Publisher's note Springer Nature remains neutral with regard to jurisdictional claims in published maps and institutional affiliations.

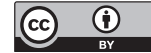

Open Access This article is licensed under a Creative Commons Attribution 4.0 International License, which permits use, sharing, adaptation, distribution and reproduction in any medium or format, as long as you give appropriate credit to the original author(s) and the source, provide a link to the Creative Commons license, and indicate if changes were made. The images or other third party material in this article are included in the article's Creative Commons license, unless indicated otherwise in a credit line to the material. If material is not included in the article's Creative Commons license and your intended use is not permitted by statutory regulation or exceeds the permitted use, you will need to obtain permission directly from the copyright holder. To view a copy of this license, visit http://creativecommons.org/ licenses/by/4.0/.

(C) The Author(s) 2021 